\title{
CONTROLE DE QUALIDADE ANGULAR EM LEVANTAMENTOS TOPOGRÁFICOS
}

\author{
Angular control quality in survey \\ JORGE PIMENTEL CINTRA \\ JEFFERSON ROCCO
}

Escola Politécnica da USP - SP - Brasil

jpcintra@usp.br; jefferson.rocco@usp.br

\begin{abstract}
RESUMO
O presente trabalho estuda o controle de qualidade de medidas angulares, que são um elemento crítico na execução de túneis metroviários e assemelhados em que é necessária a manutenção precisa de alinhamentos e das coordenadas de pontos, em uma poligonal aberta, portanto sem controle de fechamento, e com aplicação direta na orientação das máquinas de escavação e na execução do túnel. Assim, foram estudados métodos e procedimentos para controlar a precisão e a exatidão dessas medidas. Foi analisado o desempenho de diversas estações totais, em diversas campanhas; foi estabelecido um quadrilátero e seus ângulos foram medidos e ajustados, para servirem de padrão, não só para avaliar a precisão, mas também a acurácia dos equipamentos. Dos experimentos surgiram propostas, procedimentos e critérios para serem incorporadas à norma de controle de qualidade da medição de ângulos.
\end{abstract}

Palavras-chave: Controle de Qualidade Angular; Túneis Metroviários; Poligonais Abertas; Estações Totais.

\section{ABSTRACT}

This paper dealt with the quality control of angular measurements which are a critical element in the construction of subway tunnels and similar projects, in which it is necessary to maintain precise alignments and points coordinates on an open polygonal without controlled closing and with direct application on guiding digging machines and tunnel construction. Thus, methods and procedures to control the precision and accuracy of these measures were studied. Performance of several total stations, in several campaigns were analysed; a quadrilateral and their angles were 
measured and adjusted to serve as a standard, to allow not only the assessment of the precision, but also the accuracy of the equipment. As a result, proposals, procedures and criteria were presented to be incorporated into standard quality control measurement of angles.

Keywords: Quality Control Angle; Subway Tunnels; Open Traverse; Total Stations.

\section{INTRODUÇÃO}

O controle das observações feitas pelas estações totais (ou teodolitos), está diretamente associado à qualidade final dos empreendimentos de engenharia estabelecidos em projeto, desde pequenos trabalhos até grandes obras como túneis rodoviários e metroviários, galerias, minas, estradas, pontes, viadutos e tantas outras.

Dessa forma, torna-se necessário estabelecer critérios de controle da precisão e exatidão para esses equipamentos, em particular no que se refere aos ângulos. Segundo Paciléo Netto, 1993 a precisão expressa o grau de aderência das observações uma às outras, e é medida pelo desvio padrão de uma série de observações; já a exatidão expressa o grau de aderência do melhor valor (por exemplo, a média) para as observações em relação ao valor verdadeiro.

Como o valor verdadeiro não é conhecido pode-se ter uma avaliação da exatidão ou acurácia através da diferença entre a média e um valor considerado como padrão, medido com equipamento que se supõem mais preciso / exato (GIAA, 2002).

O presente trabalho propõe que se considere também a acurácia para a classificação dos equipamentos, como se faz no controle de qualidade na operação de medição de distâncias (NBR 13.133/1994), enquanto a atual norma DIN (18723) só considera a precisão (GIAA, 2002). Assim, foi implantado em campo um quadrilátero e foram determinados os ângulos padrões através das distâncias ajustadas, estabelecendo um campo de prova para a classificação de equipamentos topográficos na medição de ângulos horizontais e propondo um número de séries.

As distâncias do quadrilátero foram medidas com a ET TCA2003, que tem mostrado repetibilidade e pequeno desvio padrão, inclusive quando comparadas com as medidas padrões do campo de provas da USP, obedecendo de fato às especificações de catálogo $(1 \mathrm{~mm}+1 \mathrm{ppm})$.

Também foram realizadas diversas campanhas de medição de direções com a Estação Total TCA2003 e o Teodolito T2, na tentativa de estabelecer os ângulos padrões. $\mathrm{O}$ resultado comparativo das medidas angulares mostrou que, embora o ajustamento tenha conduzido aparentemente a bons resultados, os ângulos estabelecidos dessa forma apresentam variabilidade da ordem de 2" a 3" e assim procurou-se estabelecer esses ângulos através das distâncias medidas em um quadrilátero de precisão. 
Foram também propostos métodos, procedimentos e critérios para a classificação e controle de qualidade de equipamentos.

\section{ESTABELECIMENTO DE UM QUADRILÁTERO PADRÃO}

Na década de 1990 foi construída a Base Multipilar na raia olímpica da USP, para a calibração de equipamentos na medição de ângulos e distâncias (PACILÉO NETTO, 1997). Aproveitando quatro pilares existentes nessa base estabeleceu-se para a presente pesquisa o quadrilátero indicado na Figura 1, com distâncias dos lados superiores a $100 \mathrm{~m}$.

Figura 1 - Quadrilátero padrão estabelecido na Raia Olímpica da USP. Imagem de fundo: Google Earth (data da imagem - 2011).

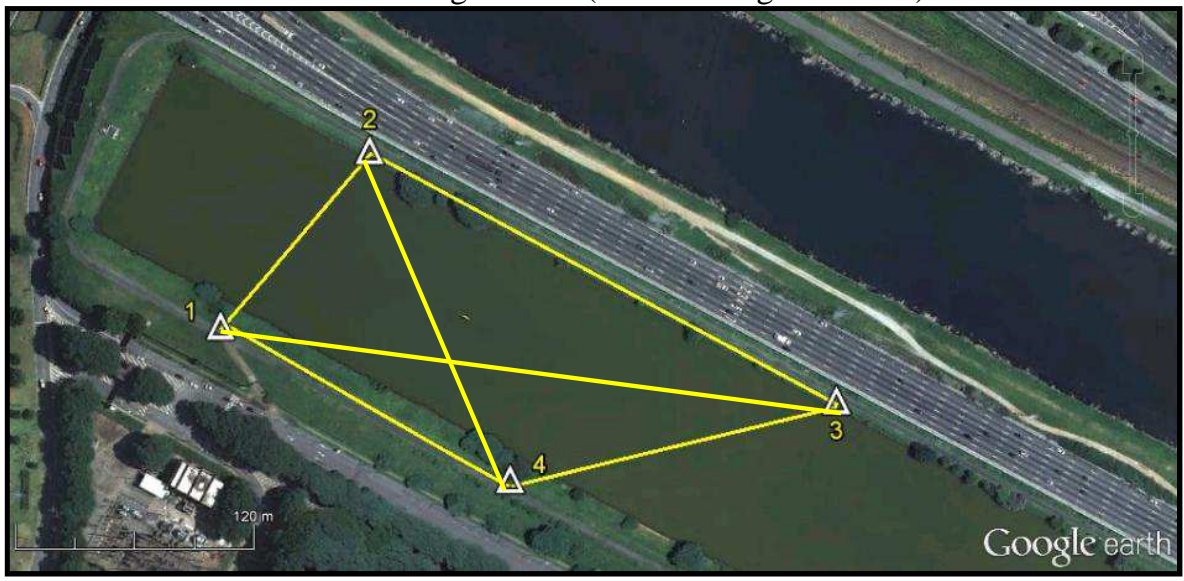

\subsection{Tentativa de Estabelecimento dos Ângulos Padrão Através de Direções}

Para determinar os ângulos padrões, foram realizadas duas campanhas com a estação total Leica TCA2003 e duas com teodolito Wild T2mod. Estes, equipamentos são considerados pela norma atual, como de alta precisão pelo valor especificado no catálogo $\left(0,5^{\prime \prime}\right.$ e $\left.1,0^{\prime \prime}\right)$, respectivamente, conforme LEICA GEOSYSTEMS (2006) e WILD HEERBRUGG (1976). Esse valor refere-se a direções; para ângulos deve-se multiplicar por $\sqrt{ } 2$, resultando em aproximadamente $0,7^{\prime \prime}$ e $1,4 "$.

Nessas campanhas foram controladas as temperaturas de bulbo seco e úmido e a pressão atmosférica; os equipamentos foram protegidos com o guarda sol e foram tomados os demais cuidados requeridos pela natureza dessa operação de estabelecimento de valores padrão, em particular séries com mais de 18 medições angulares. Para cada ângulo foi calculada a média e o desvio-padrão, através do programa Excel. 
Com os valores médios procedeu-se ao ajustamento por mínimos quadrados através do programa WolfPack (2013).

A Tabela 1 resume os resultados: para cada campanha (de 1 a 4, nas colunas) são indicados o equipamento, o ângulo ajustado por mínimos quadrados (de 1 a 8) e a somatória $(\Sigma)$, para verificação. Na última coluna figura a média de cada ângulo, considerada como resultado final.

Tabela 1 - Resumo dos ângulos ajustados e a média entre as campanhas.

\begin{tabular}{c|c|c|c|c|c}
\hline Campanha & 1 & 2 & 3 & 4 & média \\
\hline Âng/Equip & $\mathrm{T} 2$ & $\mathrm{~T} 2$ & TCA2003 & TCA2003 & \\
\hline 1 & $56^{\circ} 25^{\prime} 03,4^{\prime \prime}$ & $56^{\circ} 25^{\prime} 04,8^{\prime \prime}$ & $56^{\circ} 25^{\prime} 04,1^{\prime \prime}$ & $56^{\circ} 25^{\prime} 01,4^{\prime \prime}$ & $56^{\circ} 25^{\prime} 03,4^{\prime \prime}$ \\
\hline 2 & $20^{\circ} 46^{\prime} 34,1^{\prime \prime}$ & $20^{\circ} 46^{\prime} 32,5^{\prime \prime}$ & $20^{\circ} 46^{\prime} 33,2^{\prime \prime}$ & $20^{\circ} 46^{\prime} 33,2^{\prime \prime}$ & $20^{\circ} 46^{\prime} 33,3^{\prime \prime}$ \\
\hline 3 & $64^{\circ} 02^{\prime} 41,0^{\prime \prime}$ & $64^{\circ} 02^{\prime} 39,9^{\prime \prime}$ & $64^{\circ} 02^{\prime} 38,5^{\prime \prime}$ & $64^{\circ} 02^{\prime} 43,6^{\prime \prime}$ & $64^{\circ} 02^{\prime} 40,8^{\prime \prime}$ \\
\hline 4 & $38^{\circ} 37^{\prime} 18,4^{\prime \prime}$ & $38^{\circ} 37^{\prime} 18,4^{\prime \prime}$ & $38^{\circ} 37^{\prime} 19,8^{\prime \prime}$ & $38^{\circ} 37^{\prime} 18,8^{\prime \prime}$ & $38^{\circ} 37^{\prime} 18,9^{\prime \prime}$ \\
\hline 5 & $20^{\circ} 54^{\prime} 57,2^{\prime \prime}$ & $20^{\circ} 54^{\prime} 56,9^{\prime \prime}$ & $20^{\circ} 54^{\prime} 57,6^{\prime \prime}$ & $20^{\circ} 54^{\prime} 56,2^{\prime \prime}$ & $20^{\circ} 54^{\prime} 57,0^{\prime \prime}$ \\
\hline 6 & $20^{\circ} 07^{\prime} 28,0^{\prime \prime}$ & $20^{\circ} 07^{\prime} 24,8^{\prime \prime}$ & $20^{\circ} 07^{\prime} 24,9^{\prime \prime}$ & $20^{\circ} 07^{\prime} 26,7^{\prime \prime}$ & $20^{\circ} 07^{\prime} 26,1^{\prime \prime}$ \\
\hline 7 & $38^{\circ} 45^{\prime} 41,5^{\prime \prime}$ & $38^{\circ} 45^{\prime} 42,8^{\prime \prime}$ & $38^{\circ} 45^{\prime} 44,2^{\prime \prime}$ & $38^{\circ} 45^{\prime} 41,8^{\prime \prime}$ & $38^{\circ} 45^{\prime} 42,6^{\prime \prime}$ \\
\hline 8 & $100^{\circ} 20^{\prime} 16,4^{\prime \prime}$ & $100^{\circ} 20^{\prime} 19,9^{\prime \prime}$ & $100^{\circ} 20^{\prime} 17,7^{\prime \prime}$ & $100^{\circ} 20^{\prime} 18,3^{\prime \prime}$ & $100^{\circ} 20^{\prime} 18,1^{\prime \prime}$ \\
\hline$\Sigma$ & $360^{\circ} 00^{\prime} 00,0^{\prime \prime}$ & $360^{\circ} 00^{\prime} 00,0^{\prime \prime}$ & $360^{\circ} 00^{\prime} 00,0^{\prime \prime}$ & $360^{\circ} 00^{\prime} 00,0^{\prime \prime}$ & \\
\hline
\end{tabular}

Para análise dos resultados, na Tabela 2 está indicado o valor do desvio $(\sigma)$ de cada ângulo nas respectivas campanhas, após o ajustamento com o programa WolfPack. Nas duas últimas linhas estão indicados a média dos desvios $(\mu)$ e o desvio-padrão nominal ( $\sigma$ nom). Como se pode notar, há diferenças significativas entre os valores de um mesmo ângulo, medido pelo mesmo equipamento, em dias diferentes. Isso se deve às diferentes condições climáticas de campo: intensidade do sol e do vento, reverberação, umidade, etc. Isso apesar das precauções que se tomaram: guarda-sol, espera de condições melhores, eliminar erros grosseiros, etc.

O melhor resultado foi o da campanha 1 (T2), com média dos erros de 0,44" (em valor absoluto) e os outros estão com valores entre 0,84 " e 1,21" o que parece bastante razoável comparando com a precisão nominal para ângulos de cada equipamento: 0,71" para a ET TCA2003 e 1,41" para o teodolito T2.

Ainda na Tabela 1, comparando em cada linha os valores de cada ângulo com a média, pode-se calcular e ver que há valores que fogem mais de 2" da média, como por exemplo o ângulo 3 nas campanhas 3 e 4, além de outros; isso apesar de serem valores resultantes de uma série de medidas e que passaram por um ajustamento de mínimos quadrados no quadrilátero.

No cálculo da média da Tabela 1, a média tomada foi simples, sem pesos. Poder-se-ia pensar em uma média ponderada, em função do desvio padrão nominal (TCA2003, duas vezes melhor que o T2), ou melhor ainda, através dos desvios efetivos da Tabela 2 (que mostra o T2 mais preciso que a TCA2003). Mas, tendo em conta a magnitude das discrepâncias, optou-se pela média simples e por procurar 
outra maneira de estabelecer ângulos padrões através de outro caminho: calculados através de distâncias.

Tabela 2 - Desvios-padrão dos ângulos após o ajustamento nas 4 campanhas.

\begin{tabular}{|c|c|c|c|c|}
\hline Campanha & 1 & 2 & 3 & 4 \\
\hline Ângulo/Equipamento & $\mathrm{T} 2$ & $\mathrm{~T} 2$ & TCA2003 & TCA2003 \\
\hline 1 & $0,50 "$ & $0,94 "$ & $1,40^{\prime \prime}$ & $0,61 "$ \\
\hline 2 & $0,42 "$ & $0,74 "$ & $0,76 "$ & $0,80 "$ \\
\hline 3 & $0,38^{\prime \prime}$ & $0,58 "$ & $1,16^{\prime \prime}$ & $0,89^{\prime \prime}$ \\
\hline 4 & $0,60 "$ & $0,87 "$ & $1,00^{\prime \prime}$ & $1,11 "$ \\
\hline 5 & $0,40 "$ & $0,82 "$ & $1,12 "$ & $0,85 "$ \\
\hline 6 & $0,31 "$ & $0,56 "$ & $0,95 "$ & $0,99 "$ \\
\hline 7 & $0,44 "$ & $1,17 "$ & $1,62 "$ & $1,03 "$ \\
\hline 8 & $0,47 "$ & $1,00^{\prime \prime}$ & $1,63 "$ & $1,01 "$ \\
\hline$\mu$ & $0,44 "$ & $0,84 "$ & $1,21 "$ & $0,91 "$ \\
\hline jnom & $1,41 "$ & $1,41^{\prime \prime}$ & $0,71^{\prime \prime}$ & $0,71^{\prime \prime}$ \\
\hline
\end{tabular}

\subsection{Estabelecimento dos Ângulos Padrão Através das Distâncias}

Como alternativa para o estabelecimento dos ângulos padrões foram medidas e ajustadas as distâncias do quadrilátero: quatro lados e duas diagonais; esperando que a rigidez da figura e o ajustamento por mínimos quadrados conduzissem a resultados melhores, o que de fato ocorreu.

A estação total utilizada na presente pesquisa, ET Leica TCA2003, mostrou repetibilidade e pequeno desvio-padrão nas medidas de distâncias, quando comparadas com as medidas padrão do campo de provas da USP, obedecendo de fato às especificações de catálogo $(1 \mathrm{~mm}+1 \mathrm{ppm})$. Pela experiência, sabe-se que em geral os equipamentos possuem valores dessas duas constantes até melhores do que as do catálogo. Por outro lado, se houvesse um erro sistemático, expresso pelo coeficiente das ppm, as distâncias ficariam multiplicadas por esse valor (ampliação ou redução da figura), mas os ângulos do quadrilátero não se alterariam pois a proporção dos lados seria mantida.

Assim, foram efetuadas campanhas de medição das distâncias, que tiveram como objetivo imediato estabelecer os comprimentos dos lados do quadrilátero. Foram empregados 3 prismas de precisão, psicrômetro, barômetro, suporte metálico e guarda-sol, como nas outras campanhas. As observações, em cada campanha, foram realizadas no período da manhã e no da tarde, com a presença de sol e ausência de vento, possibilitando boa pontaria no uso da busca automática de alvo.

Foram feitas nove séries completa nas medidas das direções horizontais (PDPI), e outros cuidados foram: controle do nível da base do prisma em todas as mudanças do instrumento; controle da direção do prisma para a leitura da ET; coleta de dados automática para evitar erros de anotação.

Na Tabela 3 estão indicadas as médias das distâncias de 36 medidas de campo e os respectivos desvios de uma medida singular $(\sigma)$ e da média $(\sigma m)$. Com os 
valores médios foi feito um ajuste de quadrilátero através do programa WolfPack (2013). Na Tabela 4 apresentam-se os valores dessas distâncias ajustadas.

Tabela 3 - Distância média, desvio-padrão e desvio-padrão da média.

\begin{tabular}{c|c|c|c}
\hline Lado & Distância $(\mathrm{m})$ & $\sigma(\mathrm{mm})$ & $\sigma \mathrm{m}(\mathrm{mm})$ \\
\hline 14 & 165,1846 & 0,2 & 0,03 \\
\hline 13 & 314,3459 & 0,4 & 0,07 \\
\hline 12 & 115,0187 & 0,4 & 0,07 \\
\hline 34 & 170,2997 & 0,6 & 0,10 \\
\hline 42 & 179,1439 & 0,3 & 0,05 \\
\hline 32 & 268,4116 & 0,1 & 0,02 \\
\hline
\end{tabular}

Tabela 4 - Distâncias ajustadas dos lados do quadrilátero.

\begin{tabular}{c|c}
\hline Campanha & 1 \\
\hline & distância $(\mathrm{m})$ \\
\hline 14 & 165,1846 \\
\hline 13 & 314,3460 \\
\hline 12 & 115,0187 \\
\hline 34 & 170,2996 \\
\hline 42 & 179,1439 \\
\hline 32 & 268,4116 \\
\hline
\end{tabular}

Esse mesmo programa fornece as coordenadas dos vértices do quadrilátero, no sistema de coordenadas escolhido pelo usuário. No caso, estabeleceram-se as coordenadas $(0,0 ; 0,0)$ em metros para o ponto 1 e o eixo x segundo a direção 14 . Essas coordenadas figuram na Tabela 5.

Tabela 5 - Coordenadas ajustadas.

\begin{tabular}{c|c|c}
\hline Vértice & \multicolumn{2}{|c}{ Coordenadas } \\
\hline & $\mathrm{X}(\mathrm{m})$ & $\mathrm{Y}(\mathrm{m})$ \\
\hline 1 & 000,0000 & 000,0000 \\
\hline 2 & 25,4948 & 112,1575 \\
\hline 3 & 293,9056 & 111,5028 \\
\hline 4 & 165,1846 & 000,0000 \\
\hline
\end{tabular}

A partir dessas coordenadas calcularam-se os ângulos a partir do eixo y desse sistema $(\operatorname{tg} \mathrm{A}=\Delta \mathrm{x} / \Delta \mathrm{y}$; Tabela 6 ) onde 1-4 é a direção $\mathrm{x}$ (em função das coordenadas y serem zero). A seguir foram calculados os ângulos do quadrilátero (Tabela 7), por diferença entre as direções. Esses valores foram considerados como padrão estabelecido. 
Tabela 6 - Direção entre os vértices do quadrilátero.

\begin{tabular}{c|c}
\hline Vértice & Direção \\
\hline $1-2$ & $12^{\circ} 48^{\prime} 23,2^{\prime \prime}$ \\
\hline $1-3$ & $69^{\circ} 13^{\prime} 26,8^{\prime \prime}$ \\
\hline $1-4$ & $90^{\circ} 00^{\prime} 00,0^{\prime \prime}$ \\
\hline $2-3$ & $90^{\circ} 08^{\prime} 23,1^{\prime \prime}$ \\
\hline $4-3$ & $49^{\circ} 05^{\prime} 58,9^{\prime \prime}$ \\
\hline $4-2$ & $308^{\circ} 45^{\prime} 40,0^{\prime \prime}$ \\
\hline
\end{tabular}

Para a classificação dos equipamentos foi usado o ângulo 1 , ou seja, o vértice 1 usado para estacionar o equipamento, o 2 como ré e o 3 como vante. $\mathrm{O}$ desvio padrão foi calculado através da tangente do ângulo formado pelo semi-eixo da elipse de erro (aproximadamente perpendicular à linha de visada) e pelo comprimento do lado, resultando em $0,36 "$.

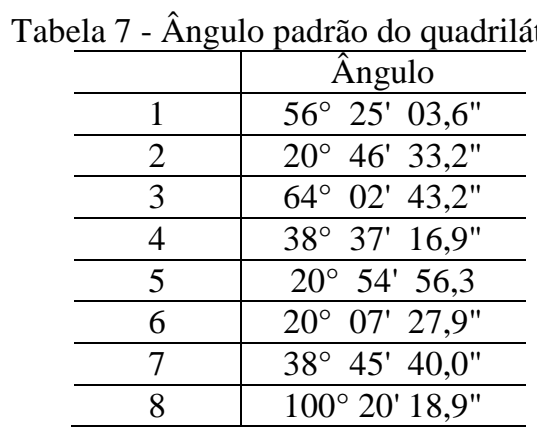

\section{CLASSIFICAÇÃO DE EQUIPAMENTOS DE MEDIÇÃO DE ÂNGULOS}

\subsection{Definições Preliminares para os Experimentos}

Contando com esses ângulos considerados como padrão foi possível testar equipamentos, não só quanto à precisão, como preconiza a NBR 13.133/1994 e a DIN 18.123, mas também quanto à acurácia.

Foram realizados 13 experimentos com equipamentos de precisão baixa, média e alta, segundo a precisão nominal fornecida pelo catálogo e as faixas indicadas na NBR 13.133/1994. Os cálculos dos ângulos médios e desvios-padrão foram realizados usando as duas posições da luneta (PD e PI).

No experimento 1 , com o equipamento GDM 600, foram ocupados todos os vértices do quadrilátero e, em cada um, a direção observada foi incrementada de $30^{\circ} \mathrm{em} 30^{\circ}$. Os resultados estão na Tabela 8 . Como se pode ver, a precisão (desviopadrão) variou relativamente pouco (de 1,8" e 3,6"), quando comparado com as faixas de classificação (Quadro 3). Isso permitiu simplificar a metodologia, ocupando daí em diante somente um vértice (o de n. 1). Ou seja, nos experimentos 
seguintes foi ocupado apenas um vértice e foram realizadas as observações de duas direções (1-2 e 1-3), nas posições direta e inversa, obtendo-se o ângulo (1), com incremento angular de $20^{\circ} \mathrm{em} 20^{\circ}$ até completar o giro de $180^{\circ}$.

Coisa semelhante foi feita com os demais equipamentos, e verificou-se que, para todos eles, o desvio-padrão apresentou pequena variação, como aconteceu com o equipamento dessa Tabela, independentemente do vértice ocupado e do ângulo medido. Isso sugere que a norma pode ser simplificada, propondo-se a medição de 2 direções somente (resultando em um ângulo), em 6 séries e não 5 direções em 4 séries. O que resulta até em menor esforço de campo.

Tabela 8 - Ângulos médios com seu desvio $(\sigma)$, Ângulos padrões e diferenças (t) para o padrão, no experimento 1, com a estação GDM 600, 6 séries conjugadas.

\begin{tabular}{c|c|c|c|c}
\hline Ângulos & $\begin{array}{c}\text { Ângulos } \\
\text { médios }\end{array}$ & $\sigma$ & $\begin{array}{c}\text { Ângulos } \\
\text { padrões }\end{array}$ & $\mathrm{t}$ \\
\hline 1 & $56^{\circ} 25^{\prime} 08,4^{\prime \prime}$ & $3,2^{\prime \prime}$ & $56^{\circ} 25^{\prime} 03,6^{\prime \prime}$ & $4,8^{\prime \prime}$ \\
\hline 2 & $20^{\circ} 46^{\prime} 32,3^{\prime \prime}$ & $3,6^{\prime \prime}$ & $20^{\circ} 46^{\prime} 33,2^{\prime \prime}$ & $-0,9^{\prime \prime}$ \\
\hline 3 & $64^{\circ} 02^{\prime} 39,8^{\prime \prime}$ & $1,9^{\prime \prime}$ & $64^{\circ} 02^{\prime} 43,2^{\prime \prime}$ & $-3,4^{\prime \prime}$ \\
\hline 4 & $38^{\circ} 37^{\prime} 15,2^{\prime \prime}$ & $3,4^{\prime \prime}$ & $38^{\circ} 37^{\prime} 16,9^{\prime \prime}$ & $-1,7^{\prime \prime}$ \\
\hline 5 & $20^{\circ} 54^{\prime} 58,2^{\prime \prime}$ & $2,5^{\prime \prime}$ & $20^{\circ} 54^{\prime} 56,3^{\prime \prime}$ & $1,9^{\prime \prime}$ \\
\hline 6 & $20^{\circ} 07^{\prime} 26,4^{\prime \prime}$ & $3,1^{\prime \prime}$ & $20^{\circ} 07^{\prime} 27,9^{\prime \prime}$ & $-1,5^{\prime \prime}$ \\
\hline 7 & $38^{\circ} 45^{\prime} 39,9^{\prime \prime}$ & $1,8^{\prime \prime}$ & $38^{\circ} 45^{\prime} 40,0^{\prime \prime}$ & $-0,1^{\prime \prime}$ \\
\hline 8 & $100^{\circ} 20^{\prime} 24,1^{\prime \prime}$ & $2,4^{\prime \prime}$ & $100^{\circ} 20^{\prime} 18,9^{\prime \prime}$ & $5,2^{\prime \prime}$ \\
\hline
\end{tabular}

\subsection{Classificação Medindo Ângulos e não Direções}

Por outro lado, a classificação dos equipamentos no presente trabalho foi realizada em função do desvio-padrão do ângulo médio obtido e não com o valor de catálogo para a direção. Como na NBR 13.133/1994 a classe dos teodolitos é definida segundo o desvio-padrão de uma direção observada em duas posições da luneta (DIN 18723), foi acrescentado na última coluna do Quadro 1, o desviopadrão para o ângulo (bastando multiplicar o valor da direção por $\sqrt{2}$ ).

Quadro 1 - Classes dos equipamentos segundo o desvio-padrão da direção e do ângulo. Adaptado da NBR 13.133 (1994).

\begin{tabular}{|ccc|}
\hline $\begin{array}{c}\text { Classes de teodolitos - } \\
\text { NBR 13.133 }\end{array}$ & $\begin{array}{c}\text { Desvio-padrão } \\
\text { da direção }\end{array}$ & $\begin{array}{c}\text { Desvio-padrão } \\
\text { do ângulo }\end{array}$ \\
1 - precisão baixa & $\leq \pm 30^{\prime \prime}$ & $\leq \pm 42,4^{\prime \prime}$ \\
2- precisão média & $\leq \pm 07^{\prime \prime}$ & $\leq \pm 9,9^{\prime \prime}$ \\
3- precisão alta & $\leq \pm 02^{\prime \prime}$ & $\leq \pm 2,8^{\prime \prime}$ \\
\hline
\end{tabular}




\section{EXPERIMENTOS E ANÁLISE DOS RESULTADOS}

\subsection{Classificação Segundo Ângulos e Desvio-Padrão}

Na Tabela 9 é apresentado um resumo dos valores obtidos em todos os experimentos, realizados de acordo com as premissas do item 3. São indicando os desvios-padrão e a classificação dos equipamentos segundo o desvio-padrão da direção e do ângulo, conforme as faixas do Quadro 1. Nesta tabela a primeira coluna indica o número do experimento; a coluna 2, o modelo do equipamento; a coluna 3 o desvio-padrão nominal da direção; a coluna 4, o desvio-padrão do ângulo ( $\sigma$ nom ang) calculado em função da coluna anterior (multiplicar por raiz de 2); a coluna 5 indicada o desvio-padrão efetivo ( $\sigma e f)$ do ângulo obtido através das medidas em campo e a 6 informa se o equipamento atendeu ou não a precisão nominal do ângulo (associado a todas as condições locais durante o experimento: temperatura, pressão, clima, cuidado do operador, etc). Na coluna 7 é indicada a classificação do equipamento segundo o ângulo, conforme Quadro 1.

Tabela 9 - Resultados dos experimentos para a classificação dos equipamentos segundo o ângulo medido entre duas direções.

\begin{tabular}{|c|c|c|c|c|c|c|}
\hline Experim. & Equip. & $\begin{array}{c}\text { onom } \\
\text { dir }\end{array}$ & $\begin{array}{c}\text { onom } \\
\text { ang }\end{array}$ & $\sigma e f$ & $\begin{array}{c}\sigma e f<\sigma \text { nom } \\
\text { ang }\end{array}$ & Classif. \\
\hline 1 & GDM 600 & $4,0^{\prime \prime}$ & $5,7 "$ & $3,2^{\prime \prime}$ & sim & Média \\
\hline 2 & $\mathrm{~T} 2$ & $1,0^{\prime \prime}$ & $1,4^{\prime \prime}$ & $1,9^{\prime \prime}$ & não & Alta \\
\hline 3 & TCA2003 & $0,5^{\prime \prime}$ & $0,7^{\prime \prime}$ & $1,6^{\prime \prime}$ & não & Alta \\
\hline 4 & TCA2003 & $0,5^{\prime \prime}$ & $0,7^{\prime \prime}$ & $1,1^{\prime \prime}$ & não & Alta \\
\hline 5 & GTS 213 & $10,0^{\prime \prime}$ & $14,1^{\prime \prime}$ & $2,9^{\prime \prime}$ & $\operatorname{sim}$ & Média \\
\hline 6 & NPR 352 & $5,0^{\prime \prime}$ & $7,1^{\prime \prime}$ & $1,5^{\prime \prime}$ & $\operatorname{sim}$ & Alta \\
\hline 7 & TC 307 & $7,0 "$ & $9,9^{\prime \prime}$ & $5,0^{\prime \prime}$ & $\operatorname{sim}$ & Média \\
\hline 8 & TC 305 & $5,0 "$ & $7,1^{\prime \prime}$ & $1,7^{\prime \prime}$ & $\operatorname{sim}$ & Alta \\
\hline 9 & TC 600 & $5,0 "$ & $7,1^{\prime \prime}$ & $3,9^{\prime \prime}$ & $\operatorname{sim}$ & Média \\
\hline 10 & TC 600 & $5,0^{\prime \prime}$ & $7,1^{\prime \prime}$ & $1,1^{\prime \prime}$ & $\operatorname{sim}$ & Alta \\
\hline 11 & TCA2003 & $0,5^{\prime \prime}$ & $0,7^{\prime \prime}$ & $1,6^{\prime \prime}$ & não & Alta \\
\hline 12 & TS02 & $7,0^{\prime \prime}$ & $9,9 "$ & $1,2^{\prime \prime}$ & $\operatorname{sim}$ & Alta \\
\hline 13 & T2 Metrô & $1,0 "$ & $1,4^{\prime \prime}$ & $1,6^{\prime \prime}$ & não & Alta \\
\hline
\end{tabular}

Assim, exemplificando para a Estação GDM 600 (Experimento 1), o desviopadrão para esse equipamento (3,2"), situa-se abaixo de 5,7" que é a precisão nominal para ângulos ( $4 \sqrt{2}=5,7$ ”). Ou seja, está dentro da precisão nominal, e portanto dentro das especificações. Quando comparado com as faixas de classes da NBR 13.133/1994 (Quadro 1) esse equipamento é classificado com sendo de precisão média (entre 2,8" e 9,9"). Todos os demais equipamentos foram classificados de maneira semelhante, com o resultado colocado na última coluna. 
Analisando essa tabela, observa-se que nos experimentos a estação total Leica TCA2003 e o teodolito Wild T2 com seus acessórios não atenderam à precisão nominal para as condições do conjunto operador/campo. Por outro lado, os equipamentos 1, 5, 7 e 9 foram classificados como sendo de precisão média e os demais como sendo de precisão alta, sempre na comparação com os limites do Quadro 1 (NBR), última coluna.

\subsection{Classificação Segundo Ângulos e Acurácia}

Uma vez feita a classificação, baseada no desvio-padrão das medidas, pode-se passar ao teste de comparação com um valor padrão. Como se apontou, a proposta é expressá-la através de um indicador que forneça uma medida da acurácia, entendida como o afastamento (t) do valor padrão.

Para o equipamento do experimento 1 (GDM) esses valores de $\mathrm{t}$ estão expressos na última coluna da Tabela 8 , já apresentada. Observa-se que $t$ varia na faixa de $-3,4$ " a 5,2 " e que portanto está no intervalo de um desvio-padrão do ângulo: $-5,7 " \leq \mathrm{t} \leq+5,7$ ". O valor do módulo do desvio-padrão e seus múltiplos podem ser propostos como um parâmetro para medir a magnitude da acurácia.

Seguindo esse princípio, na Tabela 10 apresenta-se o valor de t, diferença entre o ângulo 1 medido com cada equipamento, no respectivo experimento, menos o valor padrão desse ângulo: $56^{\circ} 25^{\prime} 03,6^{\prime \prime}$. Esse valor de t, como se comentou, pode ser considerado como uma medida da acurácia / exatidão. Assim, na primeira coluna é indicado o número do experimento, na coluna 2 o modelo do equipamento, na 3 , o desvio-padrão efetivo do ângulo ( $\sigma e f)$, na 4 o desvio-padrão nominal ( $\sigma a n g$ nom), na 5 a diferença t e na coluna 6 , se esse valor é menor que a precisão nominal do equipamento ou não.

Da análise da Tabela 10 pode-se confirmar que a diferença $(t)$ para o ângulo padrão é inferior à precisão nominal fornecida pelo fabricante para todos os equipamentos. Até o momento, os experimentos realizados em campo naquelas condições locais (temperatura, pressão, acessórios e operador) e com os equipamentos retificados e verificados, mostram que os equipamentos de precisão alta tendem a apresentar uma precisão pior que o valor nominal, porque são mais suscetíveis a pequenas variações das condições de campo, sendo que o valor dado pelo fabricante refere-se a condições médias de laboratório. Por sua vez alguns equipamentos de precisão baixa apresentam valores de campo melhor do que o valor nominal.

Um ponto a chamar a atenção é que o equipamento TC 600, apresentou diferentes precisões nas duas campanhas (9 e 10). São dois equipamentos diferentes e do mesmo modelo, testados no mesmo dia e nas mesmas condições climáticas, porém com operadores diferentes. Um equipamento foi classificado como de média precisão e o outro alta, indicando que o equipamento do experimento 10 encontra-se em melhores condições de uso, embora atendesse nos dois casos à precisão de catálogo. 
Tabela 10 - Desvio-padrão efetivo do ângulo, nominal e a diferença para o padrão.

\begin{tabular}{|c|c|c|c|c|c|}
\hline Experim. & Equip. & $\sigma e f$ & бang nom & $\mathrm{t}$ & $\mathrm{t} \leq$ oang nom \\
\hline 1 & GDM 600 & $3,2 "$ & $5,7 "$ & $4,8^{\prime \prime}$ & $\operatorname{sim}$ \\
\hline 2 & $\mathrm{~T} 2$ & $1,9 "$ & $1,4^{\prime \prime}$ & $0,3^{\prime \prime}$ & $\operatorname{sim}$ \\
\hline 3 & TCA2003 & $1,6 "$ & $0,7^{\prime \prime}$ & $-0,3^{\prime \prime}$ & $\operatorname{sim}$ \\
\hline 4 & TCA2003 & $1,1 "$ & $0,7^{\prime \prime}$ & $0,4 "$ & $\operatorname{sim}$ \\
\hline 5 & GTS 213 & $2,9^{\prime \prime}$ & $14,1^{\prime \prime}$ & $-0,4 "$ & $\operatorname{sim}$ \\
\hline 6 & \begin{tabular}{|l|} 
NPR 352 \\
\end{tabular} & $1,5^{\prime \prime}$ & $7,1^{\prime \prime}$ & $2,1^{\prime \prime}$ & sim \\
\hline 7 & TC 307 & $5,0^{\prime \prime}$ & $9,9 "$ & $0,2^{\prime \prime}$ & sim \\
\hline 8 & TC 305 & $1,7 "$ & $7,1^{\prime \prime}$ & $1,0^{\prime \prime}$ & $\operatorname{sim}$ \\
\hline 9 & TC 600 & $3,9^{\prime \prime}$ & $7,1^{\prime \prime}$ & $2,6^{\prime \prime}$ & sim \\
\hline 10 & TC 600 & $1,1 "$ & $7,1^{\prime \prime}$ & $0,3^{\prime \prime}$ & sim \\
\hline 11 & TCA2003 & $1,6 "$ & $0,7 "$ & $0,7^{\prime \prime}$ & $\operatorname{sim}$ \\
\hline 12 & TS02 & $1,2 "$ & $9,9 "$ & $3,0^{\prime \prime}$ & $\operatorname{sim}$ \\
\hline 13 & T2 - grado & $1,6 "$ & $1,4^{\prime \prime}$ & $0,6^{\prime \prime}$ & sim \\
\hline
\end{tabular}

Isso e o valor do desvio-padrão efetivo, em alguns casos superior à precisão nominal do fabricante levanta a questão da metodologia e das condições em que esse valor é calculado. Experiências de laboratório, com condições climáticas controladas levam a resultados melhores. Experimentos nesse sentido foram realizados na UFPR, em que se buscou adaptar os testes de campo a um laboratório utilizando colimadores. A aplicação dessa metodologia em ambiente adequado, garante a qualidade dos resultados (SILVA, M.M.S., 2008). Em campo, com reverberação, refração lateral, sol e outras interferências os valores pioram conforme verificado nos experimentos realizados. Assim, seria interessante testar os mesmos equipamentos da presente pesquisa nesse laboratório da UFPR.

Por outro lado, levanta-se indiretamente outra questão: a maior ou menor arbitrariedade das faixas definidas na classificação. Pelos valores obtidos nos experimentos (Tabela 10), verifica-se que três equipamentos $(6,8$ e 10) estão na classe de alta precisão $(\leq \pm 2,8$ "), embora o fabricante indique que são de média precisão.

Dessa forma, questionam-se os limites dessa divisão em classes. E pergunta-se se a classificação deve ser feita em laboratório ou em campo, pois os equipamentos destinam-se normalmente a trabalhos fora de laboratório. Constata-se que mesmo os melhores equipamentos, com precisão nominal de 0,7" e 1,4" (para ângulos), não atingiram esses valores em campo.

Parece pois interessante discutir a proposta de fixar novos limites para as classes e sugerir que sejam feitas determinações de precisão / acurácia em testes tanto de laboratório como de campo. 
Cintra, J. P. ; Rocco, J.

\section{AVALIAÇÃO DO NÚMERO DE SÉRIES NECESSÁRIAS PARA O CONTROLE DE QUALIDADE}

A NBR 13.133/1994 preconiza que sejam realizadas 4 séries completas nas duas posições da luneta, porém a presente pesquisa foi delineada para estudar também esse número de séries, para verificar a procedência ou improcedência do valor 4. Dessa forma, como análise complementar, para avaliar a variação de oef e t com o número de séries observadas, foram feitas análises tomando-se, para cada equipamento, ordenadamente $3,6,9,12,15$ e 18 séries, calculando-se para cada caso o valor de t e de oef. O resultado de t encontra-se na Tabela 11 e de oef na Tabela 12. No experimento 1 foram realizadas 6 séries e no 9, 9 séries.

Tabela 11 - Diferença t para o padrão (acurácia) em função do $\mathrm{n}^{\circ}$ de séries

Número de Séries e valor de $\mathrm{t}$

\begin{tabular}{c|c|c|c|c|c|c|c}
\hline Exp. & Equip. & 3 & 6 & 9 & 12 & 15 & 18 \\
\hline 1 & GDM 600 & $4,1^{\prime \prime}$ & $4,8^{\prime \prime}$ & - & - & - & - \\
\hline 2 & T2 & $-1,4^{\prime \prime}$ & $-0,6^{\prime \prime}$ & $0,2^{\prime \prime}$ & $0,2^{\prime \prime}$ & $0,2^{\prime \prime}$ & $0,3^{\prime \prime}$ \\
\hline 3 & TCA2003 & $-0,5^{\prime \prime}$ & $-0,5^{\prime \prime}$ & $-0,4^{\prime \prime}$ & $0,0^{\prime \prime}$ & $-0,2^{\prime \prime}$ & $-0,3^{\prime \prime}$ \\
\hline 4 & TCA2003 & $0,3^{\prime \prime}$ & $0,8^{\prime \prime}$ & $0,8^{\prime \prime}$ & $0,4^{\prime \prime}$ & $0,4^{\prime \prime}$ & $0,4^{\prime \prime}$ \\
\hline 5 & GTS 213 & $0,6^{\prime \prime}$ & $0,2^{\prime \prime}$ & $0,3^{\prime \prime}$ & $0,6^{\prime \prime}$ & $0,1^{\prime \prime}$ & $-0,4^{\prime \prime}$ \\
\hline 6 & NPR 352 & $0,6^{\prime \prime}$ & $1,7^{\prime \prime}$ & $1,5^{\prime \prime}$ & $1,8^{\prime \prime}$ & $1,9^{\prime \prime}$ & $2,1^{\prime \prime}$ \\
\hline 7 & TC 307 & $0,9^{\prime \prime}$ & $2,7^{\prime \prime}$ & $1,6^{\prime \prime}$ & $0,8^{\prime \prime}$ & $1,2^{\prime \prime}$ & $0,2^{\prime \prime}$ \\
\hline 8 & TC 305 & $2,2^{\prime \prime}$ & $1,5^{\prime \prime}$ & $0,7^{\prime \prime}$ & $0,6^{\prime \prime}$ & $1,0^{\prime \prime}$ & $1,0^{\prime \prime}$ \\
\hline 9 & TC 600 & $-0,3^{\prime \prime}$ & $1,7^{\prime \prime}$ & $2,6^{\prime \prime}$ & - & - & - \\
\hline 10 & TC 600 & $-0,1^{\prime \prime}$ & $-0,3^{\prime \prime}$ & $0,2^{\prime \prime}$ & $0,0^{\prime \prime}$ & $0,2^{\prime \prime}$ & $0,3^{\prime \prime}$ \\
\hline 11 & TCA2003 & $0,1^{\prime \prime}$ & $0,2^{\prime \prime}$ & $0,6^{\prime \prime}$ & $1,0^{\prime \prime}$ & $1,0^{\prime \prime}$ & $0,7^{\prime \prime}$ \\
\hline 12 & TS02 & $2,2^{\prime \prime}$ & $2,6^{\prime \prime}$ & $2,9^{\prime \prime}$ & $2,9^{\prime \prime}$ & $2,9^{\prime \prime}$ & $3,0^{\prime \prime}$ \\
\hline 13 & T2 Metrô & $1,6^{\prime \prime}$ & $0,9^{\prime \prime}$ & $0,6^{\prime \prime}$ & $0,4^{\prime \prime}$ & $0,6^{\prime \prime}$ & $0,6^{\prime \prime}$ \\
\hline
\end{tabular}

Tabela 12 - Desvio-padrão efetivo ( $\sigma e f)$ em função do $n^{\circ}$ de séries

\begin{tabular}{|c|c|c|c|c|c|c|c|}
\hline & & \multicolumn{6}{|c|}{ Número de Séries e valor de oef } \\
\hline Exp. & Equip. & 3 & 6 & 9 & 12 & 15 & 18 \\
\hline 1 & GDM 600 & $2,8^{\prime \prime}$ & $3,2 "$ & - & - & - & - \\
\hline 2 & $\mathrm{~T} 2$ & $1,0 "$ & $1,7^{\prime \prime}$ & $2,2 "$ & $2,1 "$ & $1,8^{\prime \prime}$ & $1,9^{\prime \prime}$ \\
\hline 3 & TCA2003 & $0,6 "$ & $1,8^{\prime \prime}$ & $1,4 "$ & $1,5 "$ & $1,7^{\prime \prime}$ & $1,6^{\prime \prime}$ \\
\hline 4 & TCA2003 & $0,7^{\prime \prime}$ & $1,1^{\prime \prime}$ & $1,0^{\prime \prime}$ & $1,2 "$ & $1,1 "$ & $1,1^{\prime \prime}$ \\
\hline 5 & GTS 213 & $2,9 "$ & $3,1 "$ & $2,8 "$ & $2,7 "$ & $3,0^{\prime \prime}$ & $2,9^{\prime \prime}$ \\
\hline 6 & NPR 352 & $1,6^{\prime \prime}$ & $1,7 "$ & $1,4^{\prime \prime}$ & $1,4^{\prime \prime}$ & $1,3^{\prime \prime}$ & $1,5^{\prime \prime}$ \\
\hline 7 & TC 307 & $1,8^{\prime \prime}$ & $2,7 "$ & $3,5^{\prime \prime}$ & $3,6 "$ & $3,9 "$ & $5,0^{\prime \prime}$ \\
\hline 8 & TC 305 & $1,0^{\prime \prime}$ & $1,5^{\prime \prime}$ & $1,9^{\prime \prime}$ & $1,7^{\prime \prime}$ & $1,8^{\prime \prime}$ & $1,7^{\prime \prime}$ \\
\hline 9 & TC 600 & $4,0^{\prime \prime}$ & $3,5^{\prime \prime}$ & $3,9^{\prime \prime}$ & - & - & - \\
\hline 10 & TC 600 & $1,8^{\prime \prime}$ & $1,2^{\prime \prime}$ & $1,1^{\prime \prime}$ & $1,1^{\prime \prime}$ & $1,1^{\prime \prime}$ & $1,1^{\prime \prime}$ \\
\hline 11 & TCA2003 & $1,5^{\prime \prime}$ & $1,3^{\prime \prime}$ & $1,4^{\prime \prime}$ & $1,5^{\prime \prime}$ & $1,4^{\prime \prime}$ & $1,6^{\prime \prime}$ \\
\hline 12 & TS02 & $1,0^{\prime \prime}$ & $1,0^{\prime \prime}$ & $1,0^{\prime \prime}$ & $1,4^{\prime \prime}$ & $1,3^{\prime \prime}$ & $1,2^{\prime \prime}$ \\
\hline 13 & T2 Metrô & $1,8^{\prime \prime}$ & $2,3 "$ & $1,9 "$ & $1,7^{\prime \prime}$ & $1,7^{\prime \prime}$ & $1,6^{\prime \prime}$ \\
\hline
\end{tabular}




\section{PROPOSTAS}

De acordo com os diversos experimentos de campo:

1) Sugere-se realizar, sujeita a outros experimentos adicionais, e de forma complementar à precisão nominal, a avaliação dos equipamentos também através da acurácia t entre o ângulo medido em campo e o ângulo padrão, a ser feita em um campo de prova, como o do quadrilátero estabelecido no presente trabalho. A deficiência do método clássico é que o valor de $\sigma_{\text {ang nom }}$ e mesmo o valor de $\sigma_{\text {ef }}$ não significam que haverá um erro dessa ordem de grandeza na medição do ângulo (e portanto da poligonal de apoio a obras em que está inserido) ou seja, esses indicadores, embora importantes, não são decisivos. No entanto, um alto valor de t leva a erro tanto no ângulo quanto na respectiva poligonal de que faz parte. Por exemplo, o equipamento TC307 (n. 7, Tabela 9), pelo indicador de precisão efetiva $\left(5,0^{\prime \prime}\right)$ poderia ter erros esperados de elevada magnitude no levantamento de uma poligonal, piores do que o equipamento n. 1 (3,2" nessa mesma Tabela 9). No entanto, o indicador t (Tabela 10) aponta o contrário: que esse equipamento 7 apresenta um erro de 0,2 ", e o equipamento 1 , apresenta um erro de 4,8". Na escolha de qual equipamento utilizar em determinado trabalho, seria importante guiar-se também pelo valor de t, além de observar, como é lógico, outros fatores: robustez, durabilidade, autonomia da bateria, comunicação com outros dispositivos, preço, etc.

2) Sugere-se como alternativa para o nome das classes de equipamentos não os títulos de alta, média e baixa, mas A, B, C, por analogia com os padrões de carta do IBGE e porque, além disso, o termo baixa é depreciativo, e o equipamento pode atender bem às necessidades do trabalho/usuário.

3) Propõe-se também que os valores de $\sigma$ dos equipamentos, nos catálogos, sejam apresentados também para ângulos e não só para direções, pois normalmente medem-se direções e determinam-se os ângulos em funções das direções, sendo esses ângulos o produto final para o usuário, e este pode ser induzido a pensar que $o$ valor fornecido é para ângulo.

4) Propõe-se que os testes para definir as classes sejam realizados em laboratório e também de campo, assim como se faz com os Medidores Eletrônicos de Distância. Para isso deve-se contar com um ângulo padrão, para comparação.

5) Parece interessante propor novos limites, de acordo com os desvios fornecidos pelos fabricantes, isso porque a função de uma classificação é separar em classes os equipamentos disponíveis e os limites atuais não realizam essa separação, porque os equipamentos vêm se tornando mais precisos.

6) Discutir novas faixas para a classificação em laboratório (e depois para campo). Em função dos resultados da Tabela 10 , coluna $4\left(\sigma_{\text {ang nom }}\right)$, propõem-se novos limites no Quadro 2, a seguir.

Com esses parâmetros, simulando a aplicação desses limites aos equipamentos analisados teríamos: Classe A: TCA2003 e T2; classe B: GDM600, NPR352, TC600; classe C: GTS213, TC307 e TS02. 
Quadro 2 - Proposta de classes de equipamentos em função do desvio padrão da direção e do ângulo obtido em laboratório.

\begin{tabular}{|ccc|}
\hline $\begin{array}{c}\text { Classes de } \\
\text { equipamentos }\end{array}$ & $\begin{array}{c}\text { Desvio-padrão da } \\
\text { direção }\end{array}$ & $\begin{array}{c}\text { Desvio-padrão do } \\
\text { ângulo }\end{array}$ \\
A & $\sigma \leq 1,5^{\prime \prime}$ & $\sigma \leq 2,0^{\prime \prime}$ \\
B & $1,5^{\prime \prime}<\sigma \leq 5,0^{\prime \prime}$ & $2,0^{\prime \prime}<\sigma \leq 7,0^{\prime \prime}$ \\
C & $5,0^{\prime \prime}<\sigma \leq 10,0^{\prime \prime}$ & $7,0^{\prime \prime}<\sigma \leq 15,0^{\prime \prime}$ \\
D & $\sigma>10,0^{\prime \prime}$ & $\sigma>15,0^{\prime \prime}$ \\
\hline
\end{tabular}

7) Sugere-se também, novas faixas para a classificação em campo, conforme indicado no Quadro 3). Em campo os desvios-padrão efetivos dos equipamentos situam-se em uma faixa muito estreita (em torno 1,5") independentemente da precisão nominal atribuída pelo fabricante ser média ou alta. Com a nova proposta isso não ocorre.

Quadro 3 - Proposta de classes de equipamentos em função da diferença entre a média e o ângulo padrão $(\mathrm{t})$.

\begin{tabular}{|cc|}
\hline $\begin{array}{c}\text { Classes de } \\
\text { equipamentos }\end{array}$ & $\begin{array}{c}\text { Diferença t (em campo) } \\
\text { para o ângulo padrão } \\
\text { A }\end{array}$ \\
$\sigma \leq 1,0^{\prime \prime}$ \\
B & $1,0^{\prime \prime}<\sigma \leq 3,0^{\prime \prime}$ \\
C & $3,0^{\prime \prime}<\sigma \leq 7,0^{\prime \prime}$ \\
D & $\sigma>7,0^{\prime \prime}$ \\
\hline
\end{tabular}

Simulando a aplicação desses limites aos equipamentos analisados teríamos: Classe A: T2, TCA2003, GTS213, TC307 e TC600 (experimento 10); classe B: NPR 352, TC305 e TC600 (experimento 9); classe C: GDM600 e TS02.

8) Que numa revisão da norma de classificação de equipamentos sejam especificadas 6 séries de medidas conjugadas, pois o resultado não varia com um número de séries maior. A NBR 13.133/1994 preconiza que as observações sejam feitas em cinco séries completas em duas direções da luneta, nos experimentos realizados, observa-se que com 6 séries de medidas conjugadas na PD e PI da luneta são suficientes para a qualidade das observações. Apesar da proposta de aumentar o número de séries, diminui o número observações e portanto o tempo gasto para a realização dos trabalhos em campo, em função de medir apenas um ângulo (duas direções). 


\section{CONCLUSÃO}

O presente trabalho estabeleceu um campo de provas preciso para a verificação e classificação de Estações Totais e Teodolitos, quanto à medição de ângulos horizontais. A tentativa de estabelecer os ângulos padrões através de medições angulares mostrou-se menos precisa (na faixa de 1" a 2", Tabela 2) do que o estabelecimento dos mesmos através de um quadrilátero em que as distâncias são medidas e os ângulos são calculados (precisão de 0,36", item 2.2).

Um amplo conjunto de experimentos, com diversos equipamentos e 18 séries para cada um, variando a posição do limbo, permitiu concluir que:

a) O desvio padrão de um equipamento é basicamente o mesmo, independentemente do ângulo que foi medido (8 ângulos do quadrilátero, para cada equipamento); com o que se sugere que, contrariamente ao que preconiza a norma atual, não é necessário medir 5 direções, mas bastam duas;

b) A classificação dos equipamentos pode ser feita também através de ângulos, que em muitos casos são o elemento básico utilizado pelo engenheiro, como por exemplo, ângulo interno de uma poligonal. Para isso, o desvio-padrão efetivo do catálogo (válido para direções) deve ser multiplicado por raiz de 2.

c) Uma verificação complementar interessante é a da acurácia de um equipamento. Enquanto a verificação da precisão mede uma consistência interna do equipamento; a verificação da acurácia, através do parâmetro t proposto, fornece uma indicação do afastamento da posição "real". Para isso foi necessário estabelecer os ângulos "padrões" no campo de prova, através de um quadrilátero com ângulos avaliados com precisão e acurácia.

d) As medições e avaliações em campo são possíveis, complementando aquilo que já se faz em laboratório. E, por sua própria natureza, simulam melhor os erros que acontecem nos trabalhos de engenharia, sujeitos a muitas vicissitudes. A comparação com os testes de laboratório é desejável e pode ser objeto de trabalhos futuros.

e) A utilização de 6 séries conjugadas (medindo duas direções em PD e PI) para a classificação de equipamentos é suficiente pois a partir desse valor a precisão e a acurácia não aumentam significativamente. O número de leituras nesse caso (6 séries $\mathrm{x} 2$ direções $\mathrm{x} 2$ posições $=24$ leituras) é inferior ao número de leituras que resulta de 4 séries e 5 direções ( 4 séries x 5 direções $\times 2$ posições $=40$ leituras) preconizadas na norma atual;

f) Uma proposta interessante é redefinir as faixas numéricas das classes atuais (alta, média e baixa) pois não estão dando conta de separar os equipamentos. Novos valores foram propostos, para as classes $(\mathrm{A}, \mathrm{B}, \mathrm{C})$.

Essas conclusões permitiram fazer algumas propostas para a oportuna incorporação em uma norma de controle de qualidade desses equipamentos, coisa que é fundamental para a realização de empreendimentos de engenharia.

\section{REFERÊNCIAS BIBLIOGRÁFICAS}


ASSOCIAÇÃO BRASILEIRA DE NORMAS TÉCNICAS. NBR 13.133: еxecução de levantamento topográfico. Rio de Janeiro, 1994. 35p.

FAGGION, P. L. Obtenção dos elementos de calibração e certificação de medidores eletrônicos de distância em campo e laboratório. Tese apresentada ao Curso de Pós-Graduação em Ciências Geodésicas da Universidade Federal do Paraná, Curitiba, 2001.

GIAA, Geomatics Industry Association of America, 2002. DIN 18723 Specification for Theodolite Accuracy. Professional Surveyor Magazine, nov. 2002. Disponível em: http://www.profsurv.com/magazine/article.aspx?i=988. Acesso em junho de 2013.

GOMES, J.P. , Determinação de desníveis de precisão utilizando Estação Total. Dissertação de Mestrado, 106p. Curso de pós-graduação em Ciências Geodésicas, Universidade Federal do Paraná. Curitiba, 2006.

LEICA GEOSYSTEMS AG. Manual técnico TPS - system 1000, versão 2.4. Heerbrugg, Suiça, 2006. 256p. Suiça

PACILÉO NETTO, N., Métodos de ajustamento em geodésia e topografia. Escola Politécnica, Universidade de São Paulo, São Paulo, 1993.

PACILÉO NETTO, N., Campo de provas para instrumentos de medição e posicionamento. 1997. 160p. Trabalho de Livre-Docente - Escola Politécnica, Universidade de São Paulo, São Paulo, 1997.

SILVA, M. M. S. Metodologia para a criação de um laboratório para classificação das componentes angulares horizontal e vertical, de teodolitos e estações totais. 2008. 139p. Tese de Doutorado. Universidade Federal do Paraná. Curitiba, Paraná, 2008.

WILD HEERBRUGG - "El teodolito y su Empleo". 1976.

WOLFPACK, versão 5.0.1. Disponível em http://surveying.wb.psu.edu/psusurv/free.htm (2013).

(Recebido em outubro de 2013. Aceito em abril de 2014). 